\title{
Session 3: Emerging technologies
}

Tuesday 17 September 2002, Moderators: Keith James

[09.00-09.20]

Selection and engineering of antibodies for human therapy

Dee Athwal

Celltech Chiroscience, Slough, UK

The intrinsic properties of antibodies has resulted in them becoming widely exploited as tools in both basic research and the treatment of human disease. This, especially the latter, has been made possible through our increased understanding of antibody structure. The sequences of thousands of antibodies has been determined, and as an early example of the use of bioinformatics, the mechanism by which antibodies are able to recognise antigens was proposed. Elucidation of the three-dimensional structure of antibodies including complexes of the antibody with its antigen, has enabled the structure / function relationship of antibodies to be determined.

This presentation will introduce the domain organisation of antibodies and how the understanding of antibody architecture, has been exploited in the design of therapeutic antibodies.

The use of naked antibodies and antibody drug conjugates will be discussed. Additionally the use, progress and limitations of antibody fragments in the clinic. Recent data on an alterative technology to the use of hybridoma technology for the selection and cloning of antibodies will also be discussed.

\section{[09.20-09.40]}

\section{The n-CoDeR ${ }^{\mathrm{TM}}$ antibody libraries: Concept and applications \\ Eskil Soderlind \\ BioInvent Therapeutic AB SE-223 70 Lund, Sweden Tel.: +46 2868577; 2868550; Fax: +46 2110806; \\ E-mail:es@bioinvent.com; URL: http://www.bioinvent.com/}

The $n-C o D e R^{\mathrm{TM}}$ antibody libraries are built by letting diverse in vivo formed complementarity determin- ing regions (CDR:s) recombine into one single master framework. These CDR:s are sampled from in vivo processed and proofread gene sequences, resulting in an optimal level of correctly folded and reactive molecules. Highly specific and functional human antibody fragments have been selected using the n$\mathrm{CoDeR}^{\mathrm{TM}}$ libraries and selected specificities can be converted to IgG-molecules with high affinities and productivity levels. The latest results will be presented.

[09.40-10.00]

\section{Virus-like particles: A versatile tool for therapeutic vaccination \\ Martin F. Bachmann \\ Cytos Biotechnology AG, Zürich-Schlieren, Switzerland}

The immune system has been educated to distinguish between non-infectious self and infectious nonself. Hence, it mounts strong immune responses upon microbial infection but usually fails to respond to self molecules. Understanding the mechanisms of this self/non-self discrimination by the immune system is critical for our ability to rationally design vaccines. In recent years, it has become evident that several factors in addition to $\mathrm{B}$ and $\mathrm{T}$ cell tolerance contribute to self/non-self discrimination. As discussed in the following, these parameters include order and repetitiveness of antigenic epitopes, local antigen distribution as well as additional parameters.

Most viruses consist of few proteins only with structural restrictions impinged on them. In fact, using these few proteins, viruses are forced to generate a quasicrystalline, highly repetitive surface. Such highly repetitive antigens efficiently cross-link B cell receptors, a process which generates strong activation signal in B cells. In contrast, self antigens are usually not highly organized, in particular not those accessible to $\mathrm{B}$ cells. Thus, B cells use antigen organization as a marker for infectious non-self. Vaccines based on virus 
like particles (VLPs) exploit this basic phenomenon, since VLPs exhibit surfaces as repetitive and organized as those of viruses. Consequently, similar to viruses, VLPs are able to trigger strong B cell responses in the absence of adjuvants.

The local antigen distribution, both in space and time, is a second critical factor influencing the magnitude of the immune responses. Antigens exhibiting an even distribution that does not change over time are usually self molecules that remain ignored by the immune system and fail to trigger an immune response. Classical high and low zone tolerance experiments may serve as illustrative examples. In contrast, antigens that are concentrated locally and exhibit sharp gradients over time are typical for infections and readily trigger immune responses. Since VLPs are particulate antigens that are injected locally, they exhibit high local concentrations that vary over time and may therefore fulfill the above stipulated criteria for efficient immunogens.

Although VLPs trigger strong antibody responses in the absence of adjuvants, $\mathrm{T}$ cell responses appear to be weaker in general, in particular if highly purified antigen preparations are used. Thus, in contrast to B cell responses, which seem to be comparable to those induced by whole viruses, $T$ cell responses induced by VLPs are weaker. Activation of $\mathrm{T}$ cells requires presentation of antigen by professional antigen presenting cells (APCs). However, since VLPs are efficiently and preferentially processed by macrophages and dendritic cells, this may not usually be the limiting factor. Other parameters that seem to be required for the induction of strong $\mathrm{T}$ cell responses by VLPs will be discussed.

[10.00-10.20]

\author{
Genetic immunization: A rapid way of developing \\ high quality diagnostic or therapeutic antibodies \\ against native proteins \\ John Thompson \\ Genovac AG, Waltershofener Str. 17, D-79111 \\ Freiburg, Germany \\ Tel.: +4976145636 0; Fax: +4976145636 29; \\ E-mail: jthompson@genovac.com; \\ URL:www.genovac.com
}

In order to validate and clinically utilize the mass of new target proteins for various diseases that are now becoming available in the post-genomic era, there is a large demand for the rapid development of highly specific reagents that recognize the native proteins. Antibodies generated by genetic immunization fulfil these requirements. An interesting cDNA is cloned directly into a eukaryotic expression vector, which is then introduced into mice or other higher animals. These animals translate the cDNA into protein which is then recognized as being foreign and thus stimulates an immune response. Polyclonal antibodies can be gained six weeks after receipt of the cDNA and used for target validation. Using the same constructs, monoclonal antibodies can then be generated against interesting targets. GENOVAC has developed and patented an assay system whereby antibodies in immune sera or hybridoma supernatants can be detected without needing to isolate the protein. This assay is based on the same expression vectors that are used for immunization. Genetic immunization technology has several important advantages over conventional immunization methods:

1. Genetic immunization is extremely fast because it involves the introduction of a cDNA into the animal to directly generate antibodies against the encoded protein. Conventional methods necessitate protein purification or peptide synthesis prior to immunization.

2. Antibodies generated by genetic immunization recognize the native protein. This is generally not the case if antibodies are produced against synthetic peptides or against recombinant proteins generated in bacteria. Genetic immunization utilizes the animal cells to present the protein, thus allowing native proteins to be made that might be impossible with conventional technologies.

3. Genetic immunization avoids the development of antibodies against co-purified contaminants. Immunogenic contaminating proteins are often present following protein purification procedures from tissue, bacterial or cell extracts.

4. Genetic immunization favours high affinity antibodies. Monoclonal antibodies developed by GENOVAC by this technology have been found to have affinities in the subnanomolar range.

5. Genetic immunization can directly generate antibodies against chosen regions of a protein. Such antibodies offer the possibility of differentiating splice variants or blocking protein function. PCR amplification is easily carried out for a given exon or a DNA region encoding a functional domain for cloning into an immunization vector to generate antibodies against these regions. It is also possible to introduce specific mutations at chosen sites, which could be useful in generating antibodies needed to differentiate strongly polymorphic sequences, e.g., viral proteins. 
Murine monoclonal antibodies can either be humanized for therapeutic purposes, e.g., by CDR grafting or the technology can be applied to immunize mice that are transgenic for the human immunoglobulin heavy and light chain gene regions. This latter technology will allow the generation of fully human antibodies for therapeutic purposes. Furthermore this will allow highaffinity, human antibodies to be generated in as little as three months starting from a cDNA.

\section{[10.20-10.40]}

Targeting mucosal sites by polymeric immunoglobulin receptor-directed peptides Kendra D. White and J. Donald Capra Oklahoma Medical Research Foundation, Oklahoma, USA

Polymeric immunoglobulins provide first line humoral defense at mucosal surfaces to which they are specifically transported by the polymeric immunoglobulin receptor (pIgR) on mucosal and glandular epithelial cells. Previous studies from our laboratory sug- gested that amino acids $402-410$ of the $\mathrm{C} \alpha 3$ domain of dimeric IgA represented a potential binding site for the pIgR. Here by binding soluble human pIgR to overlapping decapeptides of $\mathrm{C} \alpha 3$, we confirm these residues and also uncover an additional site. Furthermore, we show that the $\mathrm{C} \alpha 3$ motif appears to be sufficient to direct transport of green fluorescent protein through the pIgR-specific cellular transcytosis system. An alternative approach identified phage peptides, selected from a library by the in vitro Madin Darby Canine Kidney transcytosis assay, for pIgR-mediated transport through epithelial cells. Some transcytosis-selected peptides map to the same $402-410$ pIgR-binding C $\alpha 3$ site. Further in vivo studies document that at least one of these peptides is transported in a rat model measuring hepatic bile transport. In addition to identifying small peptides that are both bound and transported by the pIgR, this study provides evidence that the pIgR-mediated mucosal secretion system may represent a means of targeting small molecule therapeutics and genes to mucosal epithelial cells. 